\title{
Interaction between tylosin and bentonite clay from a pharmacokinetic perspective
}

\author{
Mathias Devreese, Ann Osselaere, Joline Goossens, Siegrid De Baere, Patrick De Backer*, Siska Croubels
}

Department of Pharmacology, Toxicology and Biochemistry, Faculty of Veterinary Medicine, Ghent University, Salisburylaan 133, 9820 Merelbeke, Belgium e-mail: mathias.devreese@ugent.be

Introduction

Mycotoxin binders are commonly used in the feed additive industry to prevent mycotoxicosis in farm animals (Kolosova and Stroka, 2011). These products are deemed to adsorb mycotoxins in the gut and consequently prevent mycotoxin absorption. Recently, the European Food Safety Authority (EFSA, 2010) stated that too little is known about the interaction between mycotoxin binders and veterinary drugs. Therefore more studies are needed to investigate possible interactions at the level of intestinal absorption. Some suggestions have been made about a possible interaction between macrolide antibiotics and bentonite (EFSA, 2011), however no specific proof has been put forward yet. The Canadian Bureau of Veterinary Drugs (1992) reported a case of lack of efficiency of tylosin when fed concurrently with bentonite to bovines. Shryock et al. (1994) reported that tilmicosin added to the diet in broiler chickens prevented development of air-sacculitis after inoculation with Mycoplasma gallisepticum, whereas a combined use of tilmicosin and bentonite did not prevent air-sac lesions or clinical symptoms, indicating a possible negative interaction between both. Therefore, the goal of this study was to investigate the capacity of bentonite to bind tylosin, based on specific pharmacokinetic characteristics instead of clinical symptoms which can be biased.

\section{Materials and methods}

Sixteen 3 weeks-old broiler chickens (Ross 308) were randomly allocated in two groups of 8 birds. After a one-week acclimatization period, the animals were famished for $12 \mathrm{~h}$. This was followed by an oral intra-crop bolus. One group received a bolus of tylosin ( $24 \mathrm{mg} / \mathrm{kg}$ BW, Tylan Soluble, Elanco Animal Health). The other group received a bolus of tylosin $(24 \mathrm{mg} / \mathrm{kg} \mathrm{BW})$ and bentonite $(0.1 \mathrm{~g} / \mathrm{kg} \mathrm{BW})$ (Toxisorb Classic, Süd-Chemie). At several time-points after bolus administration, blood was drawn and plasma concentrations of tylosin A were analyzed using a quantitative LC-MS/MS method (Devreese et al., 2012). Plasma concentration-time profiles were evaluated (Figure 1) and several toxicokinetic parameters were calculated (WinNonlin 6.2, Pharsight Corp., USA) and compared between groups (Table 1 ) in order to evaluate the tylosin binding capacity of bentonite clay.

Results

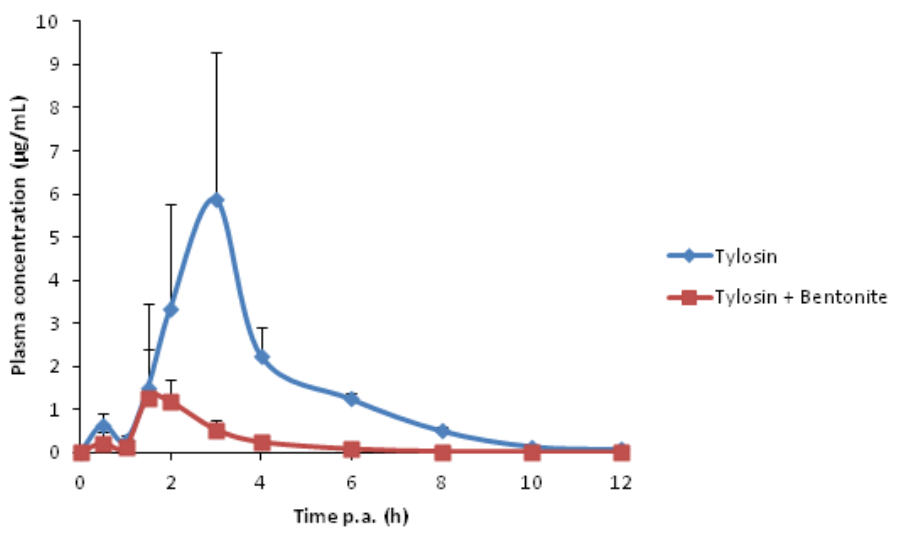

Figure 1. Plasma concentration-time profile of tylosin A after a single oral bolus administration of tylosin $(24 \mathrm{mg} / \mathrm{kg}$ BW) with or without bentonite $(0.1 \mathrm{~g} / \mathrm{kg} \mathrm{BW})$ to broiler chickens. Values are presented as mean $+\mathrm{SD}(\mathrm{n}=8)$

\begin{tabular}{|c|c|c|c|}
\hline Pharmacokinetic Parameter & Tylosin & \multicolumn{2}{|c|}{ Tylosin + Bentonite } \\
\hline AUC $_{0 \text {-inf }}(\mu \mathrm{g} \cdot \mathrm{h} / \mathrm{mL})$ & $17.06 \pm 5.31$ & $2.89 \pm 1.14$ & $* *$ \\
\hline$C_{\max }(\mu \mathrm{g} / \mathrm{mL})$ & $7.08 \pm 3.64$ & $1.66 \pm 0.77$ & $* *$ \\
\hline$T_{\max }(h)$ & $3.00 \pm 0.33$ & $1.92 \pm 0.42$ & $*$ \\
\hline$k_{e l}\left(h^{-1}\right)$ & $0.51 \pm 0.08$ & $0.59 \pm 0.23$ & \\
\hline$T_{1 / 2 \mathrm{el}}(\mathrm{h})$ & $1.42 \pm 0.21$ & $0.67 \pm 0.99$ & \\
\hline Relative OBB (\%) & & $23.3 \pm 7.22$ & $* * *$ \\
\hline \multicolumn{4}{|c|}{$\begin{array}{l}\mathrm{AUC}_{0 \text {-in: }} \text { area under plasma concentration-time profile from time } 0 \text { to infinite; } \mathrm{C}_{\max } \text { maximal plasma } \\
\text { concentration; } T_{\text {max }} \text { : time to maximal plasma concentration; } K_{\mathrm{e}}: \text { elimination rate constant; } \mathrm{T}_{1 / 2 \mathrm{el}} \text { : } \\
\text { elimination half-life; relative OBB: relative oral bioavailability; }{ }^{*} p<0.05,{ }^{* *} p<0.01 \text { and }{ }^{* * *} p<0.001\end{array}$} \\
\hline
\end{tabular}

Discussion

These results confirm the suggestion made by other authors (Canadian Bureau of Veterinary Drugs, 1992; EFSA, 2011; Shryock et al., 1994) that bentonite is able to bind macrolide antibiotics. This was demonstrated by a significant alteration of following pharmacokinetic parameters reflecting drug absorption due to the addition of bentonite to the oral bolus: area under the plasma concentration-time curve from time 0 to infinite $\left(A \cup C_{0 \text {-inf }}\right)$, maximal plasma concentration $\left(C_{\max }\right)$, time to maximal plasma concentration $\left(T_{\max }\right)$ and relative oral bioavailability (relative $\mathrm{OBB}$ ). The combined use of tylosin and bentonite in animal husbandry should therefore be avoided, as this interaction can have serious consequences. Lowered plasma concentrations of antibiotics may implicate therapy failure and enhanced microbial resistance development.

Future research should be performed on the interaction with other veterinary drugs and different mycotoxin binders. This research should be based on specific pharmacokinetic parameters instead of unspecific clinical symptoms, as only pharmacokinetic parameters can really prove a decreased oral bioavailability of the drug, due to adsorption.

\section{References}

Canadian Bureau of Veterinary Drugs, 1992. Suspected drug adverse reactions reported to The Bureau of Veterinary Drugs. Canadian Veterinary Journal 33, 237-244.

Devreese, M., Osselaere, A., Goossens, J., De Baere, S., De Backer, P., Croubels, S., 2012. Interaction between tylosin and bentonite clay from a pharmacokinetic perspective. The Veterinary Journal, In Press.

EFSA, 2010. Statement on the establishment of guidelines for the assessment of additives from the functional group substances for reduction of the contamination of feed by mycotoxins'. EFSA Journal 8, 1693.

EFSA, 2011. Scientific Opinion on the safety and efficacy of bentonite (dioctahedral montmorillonite) as feed additive for all species. EFSA Journal 9, 2007.

Kolosova, A., Stroka, J., 2011. Substances for reduction of the contamination of feed by mycotoxins: a review. World Mycotoxin Journal 4, 225-256.

Shryock, T.R., Klink, P.R., Readnour, R.S., Tonkinson, L.V., 1994. Effect of Bentonite Incorporated in a Feed Ration with Tilmicosin in the Prevention of Induced MycoplasmaGallisepticum Airsacculitis in Broiler-Chickens. Avian Diseases 38, 501-505. 